\title{
Cost Effective Data Center and its Implementation in Industry
}

\author{
Ahmed Mateen \\ Department of Computer \\ Science, \\ University of Agriculture \\ Faisalabad, Pakistan
}

\author{
Zulifqar Ali \\ Department of Computer \\ Science, \\ University of Agriculture \\ Faisalabad, Pakistan
}

\author{
Salman Afsar Awan, PhD \\ Department of Computer \\ Science, \\ University of Agriculture \\ Faisalabad, Pakistan
}

\begin{abstract}
A data Centre is a dedicated space where companies can keep and operate most of the ICT infrastructure that supports their business. A server cultivate much of the time requires expansive redundant or fortification control supply structures, cooling structures, abundance frameworks organization affiliations and methodology based security systems for running the endeavor's middle applications. This would be the servers and capacity gear that run application programming and process and store information and substance. For a few organizations this may be a basic enclosure or rack of gear, for others it could be a room lodging a couple or numerous cupboards, contingent upon the size of their operation. it divided the research in to two phases in first Phase it would be analyzed the current data center infrastructure which includes energy cost, SLA (Service Level Agreement) cost, Hardware cost, maintains cost. In second phase it is suggested how to reduce electricity cost, how to control heating in data centers, how to utilize hardware at its maximum level. It also provided better solution for security, redundancy and updation. CFD modeling tool is use for analyzing data center on generated physical test bed. Both types of analysis (qualitative and quantitative).
\end{abstract}

\section{Keywords}

Data Center, SLA (Service Level Agreement), CFD Modeling Tool

\section{INTRODUCTION}

Today, numerous substantial associations work various server farms. The purposes behind this incorporate normal business dissemination, the requirement for high accessibility and catastrophe resilience, the sheer size of their computational foundation, the craving to give uniform access times to the framework from broadly dispersed customer sites. [1] Regardless of the reason, these associations expend huge measures of vitality and this vitality utilization has both natural expense. Strikingly, the topographical dispersion of the server farms regularly uncovered numerous open doors for enhancing vitality utilization and expenses by astutely disseminating the computational workload. It is keen on three such open doors. To begin with, it try to adventure server farms that compensation distinctive and maybe variable power prices. [2] truth be told, numerous force utilities now permit customers to pick hourly evaluating, e.g. it can be look to adventure server farms that are situated in various time zones, which add an additional segment to value variability .For instance, one server farm might be under crest request costs while others are under off-top interest costs. Vitality utilization that is for the most part created via carbonconcentrated means, for example, coal red force plants.
To make the examination of these degrees of opportunity more concrete, in this paper it is consider multi-datacenter Internet administrations, for example, Google or iTunes. These administrations put their server farms behind an arrangement of front-end devices. [3] The front-closures are in charge of investigating every customer demand and sending it to one of the server farms that can serve it, as per a solicitation appropriation approach. Regardless of their widezone conveyance of solicitations, administrations must endeavor not to damage their administration level understandings (SLAs).This research proposes and assesses a structure for advancement based solicitation dissemination. [4]The system empowers administrations to deal with their vitality utilization and expenses, while regarding their SLAs. It likewise permits administrations to take full preferred standpoint of the degrees of free do said above. In light of the structure, it proposes two solicitation conveyance arrangements. For examination, it additionally propose a covetous heuristic planned with the same objectives and requirements as alternate arrangements.

Operationally, a streamlining based strategy denes the portion of the customers' demands that ought to be coordinated to every server farm. [5] The front-closes occasionally (e.g., once every hour) take care of the advancement issue by the approach. After portions are processed, the front-closes maintain them until they are recomputed. The heuristic strategy works in an unexpected way. Amid every hour, abuses the server farms with the best power effectiveness, and afterward begins misusing the server farms with the least expensive power. The assessment utilize a day-long follow from a business administration and outcomes demonstrate that the streamlining based approaches can accumulate considerable cost diminishments by wisely utilizing time zones and hourly power costs. [6] The comes about likewise demonstrate that it can misuse environmentally friendly power vitality to accomplish sign cant diminishments in chestnut vitality utilization for little increments in expense.

Late enhancements in PC and system designs have made web based applications and distributed computing frameworks mainstream. A few organizations, for example, Google, Amazon, Facebook, Microsoft, have different, geologically dispersed server farms with thousands to a huge number of servers. One vital issue of these enormous calculation arranged structures is their vitality utilization because of their huge interest. [7]A late study demonstrates that the aggregate vitality utilization of all server farms on the planet has expanded by $56 \%$ from 2005 to2010. As an aftereffect of this critical issue, there is a huge assortment of studies centering change deciphers into a great many dollars investment funds for substantial scale server farms. This subject will keep on being vital later on as the cost of chestnut vitality, the vitality 
delivered by non-renewable assets; ascend because of extra expenses put on carbon discharges. Vitality effective arrangements, extending from using environmentally friendly power vitality sources, for example, sunlight based and twist, to upgrading HW, SW and framework plan for vitality productivity, alongside crest power mindful arrangements will keep on being critical.

\section{RELATED WORK}

\subsection{Data center distribution}

Generally as a grounds correspondences room would have its own administration conveyance outline, a Distributed Patching Frame (DPF) is built up for every grounds part of the Data Center to aggregate the required availability from dynamic gadgets introduced inside the set up territory;

Which it will now term as a "portion". [8]Distributed Patching Frames is reliant upon the last division of the Data Center into its "grounds" structure. The measuring of any single DPF is reliant upon the most extreme number of cupboards that might be introduced into a "grounds" section, the greatest number of copper and fiber associations that will be required to bolster the cupboards and the DPF, and the evaluated number of switches, terminal servers, and other dynamic parts that will bolster the bureau gadgets, in addition to development. Utilizing this idea, it gets to be conceivable to extend the Data Center to another floor and not surpass the limit of the Data Center Core or disturb the movement of different portions inside the Data Center.

\subsection{Centralized processing}

is registering done at a focal area, utilizing terminals that are connected to a focal PC. The PC itself may control every one of the peripherals straightforwardly (on the off chance that they are physically associated with the focal PC), or they might be appended by means of a terminal server. [9]Alternatively, if the terminals have the capacity, they might have the capacity to interface with the focal PC over the system. The terminals might be content terminals or slender customers, for instance. It offers more prominent security over decentralized frameworks since the majority of the preparing is controlled in a focal area. Likewise, in the event that one terminal separates, the client can just go to another terminal and log in once more, and the greater part of their documents will in any case be accessible.[10] Depending on the framework, they may even have the capacity to continue their session from the point they were at some time recently, as though nothing had happened.

This sort of game plan has a few weaknesses. The focal PC plays out the figuring capacities and controls the remote terminals. This sort of framework depends absolutely on the focal PC. Should the focal PC crash, the whole framework will "go down" (i.e. will be occupied). Another inconvenience is that focal processing depends vigorously on the nature of organization and assets gave to its clients. Should the focal PC be insufficiently bolstered by any methods (e.g. size of home registries, issues with respect to organization), then your utilization will endure enormously. [11]The reverse circumstance, be that as it may, (i.e., a framework upheld superior to your requirements) is one of the key points of interest to incorporated figuring

\subsection{Hybrid server farm}

Your business requires an adaptable IT foundation that can scale on interest. With a private cloud in your datacenter, you can be more spry and oversee assets all the more adequately. When it extends your datacenter to meet the general population cloud, you are working in a half breed cloud model. A half and half cloud gives you the best of both universes, so you can exploit outer assets when it bodes well for your business. The Microsoft cross breed cloud consolidates Microsoft Azure, Windows Server, and Microsoft System Center, giving the Microsoft's endeavor grade innovation in both your organization's own datacenter and worldwide datacenters.

Stretching out into the cloud ought to grow your choices without including multifaceted nature. A Microsoft cross breed cloud lets you effectively move workloads from your datacenter to Microsoft Azure or a facilitating administration supplier's datacenter, while as yet keeping up a complete perspective of the framework. it can manufacture half and half applications that influence both on-premises and cloud assets. Also, it can exploit stockpiling, reinforcement, and recuperation choices with expanded productivity and lessened expense.

\subsection{Data center facilities}

At the most fundamental level, a server farm, including a Houston collocation office, is a building lodging server, system and capacity equipment. The server farm additionally consolidates power, cooling, fire concealment and security frameworks, and also arranges availability.

In any case, a wide range of sorts of offices and administration models exist. The accompanying rundown will illuminate the primary contrasts:

In-House Data Center - Many ventures, particularly bigger associations and those in the innovation business, outline, assemble and work their own offices. [12] The abilities of an in-house office will rely on upon the speculation the firm is willing and ready to make.

Collocation - One case of a multi-inhabitant server farm, colocation space can be sold to ventures by the rack, bureau or enclosure. Organizations of different types and sizes, from little and medium-sized organizations to Fortune 500 firms, advantage from colocation administrations. [13]Customers still keep up control over their equipment, however outsource office and inside frameworks support to the supplier.

Wholesale Data Center - These suppliers offer server farm space in bigger limits than a colocation model and regularly have less customers.[14] They may work to-suit space for a solitary occupant. The idea is like renting a distribution center or office space in which the proprietor gives office support to the occupant.

Devoted Hosting - The supplier works and/or rents server ability to single clients. At the end of the day, servers are not shared among different clients. No extra administrations are given and the client keeps up full control over the server past upkeep. Notwithstanding, a few suppliers can include enhancement administrations, for example, remote hands to re-boot servers and update programming.

Overseen Hosting - In an oversaw facilitating office, the supplier works servers and capacity for its clients, and additionally gives extra managerial and designing administrations. [15]Although the extent of administrations can be assorted and broad among the different suppliers, some basic illustrations incorporate database organization, working framework organization, oversaw security administrations, 
and oversaw capacity, application administration administrations, debacle recuperation, frameworks checking and remote administration. The equipment might be claimed by the client or the supplier.

Shared Hosting In this case, clients offer server limit. Surely understood site facilitating organizations, including Go Daddy and Network Solutions, speak to case of suppliers working shared facilitating offices. To send administrations, these suppliers make a UI overlaying the physical server. This interface gives multi-occupant applications to help clients design their administrations.

\section{PROPOSED WORK}

\subsection{Some best practices for designing cost effective data center}

\subsubsection{Spread excess over different, lower-level server farms}

Consider whether updating the IT system can decrease the office accessibility required. For instance, can three less exorbitant Tier 2 offices serve your requirements superior to anything two all the more excessive Tier 4 offices? (Level rankings depend on The Uptime Institute's level whitepaper).

The cost focuses in higher accessibility outline are:

Repetitive frameworks with excess segments in every framework

The expanded working cost those outcomes from decreased productivity because of an excess framework and parts.

Two Tier 4 offices with a four-megawatt PC burden may cost $\$ 100$ million each for an aggregate first cost of \$200 million, while three Tier 2 offices of four-megawatt PC burden may every expense $\$ 46$ million for an aggregate first cost of $\$ 138$ million. The distinction in first cost might be $\$ 62$ million. The tradeoffs need to do with system costs versus office costs. System expenses will be higher for three offices than for two.

\subsubsection{Concentrate on adaptability and measured quality}

On the off chance that the underlying UPS need is 50 watts/square foot and a definitive need is 150 watts, outline for 75 watts at first that can be later moved up to 150 watts. In the event that the underlying raised floor need is 20,000 square feet and a definitive is 40,000 square feet, plan for 30,000 square feet at first with an extra 10,000 square feet shelled in for future fit out. At the point when confronted with a decision amongst quality and amount, settle on quality that can be overhauled later on to give included amount.

I make a decent attempt and quick standards since every customer's needs are distinctive and deserving of autonomous thought. A few redesigns are easy to perform and propose proportions for thought. For instance, on Day 1 we introduce two UPS frameworks on limit N to supply N PC load in a framework + framework course of action. On Day 2 when the proprietor needs $2 \mathrm{~N}$ PC load, we can include another pair of frameworks or we can include a third arrangement of limit $\mathrm{N}$ to the two unique frameworks and reconfigure the game plan as $3 \mathrm{~N} / 2$.

\subsubsection{Keep server farm outline straightforward}

Keep the configuration as basic as could be allowed. Straightforwardness wipes out superfluous segments, and cost builds unwavering quality and viability.

Here are a few illustrations:

Minimize the quantity of layers in the electrical dispersion framework to decrease cost and make specific coordination of the electrical framework simpler.

Dispose of auxiliary chilled water pumps by making the essential chilled water pumps variable pace.

On the off chance that the greater part of the PC hardware is double line and there are no engine loads supplied by the UPS, then static exchange switches downstream of the UPS frameworks are superfluous.

\subsubsection{Stay away from exceptional or serial number 1 items}

Use standard segments and evaluations wherever conceivable. Serial number 1 dependably has a high beginning and substitution costs. For instance, 4000-ampere switchgear transport is more practical than 5000-ampere transport.

I as of late got notification from a customer that has 12-yearold UPS gear that is no more fabricated has some custom, unique control sheets. As UPS hardware ages, the accessibility of extra parts and prepared specialists diminishes.

Furthermore, robotize ordinary and crisis office operations however much as could be expected. Ultimately, use innovation rather than individuals wherever reasonable.

Take after these practices and you will be en route to outlining a savvy server farm.

3.2 Test case for measuring the temperature This experiment is set up for measure the temperature in information center. The temperature in the server farm is controlled by utilizing 5 split A.C .The temperature of the server farm is appeared beneath notice graph. 


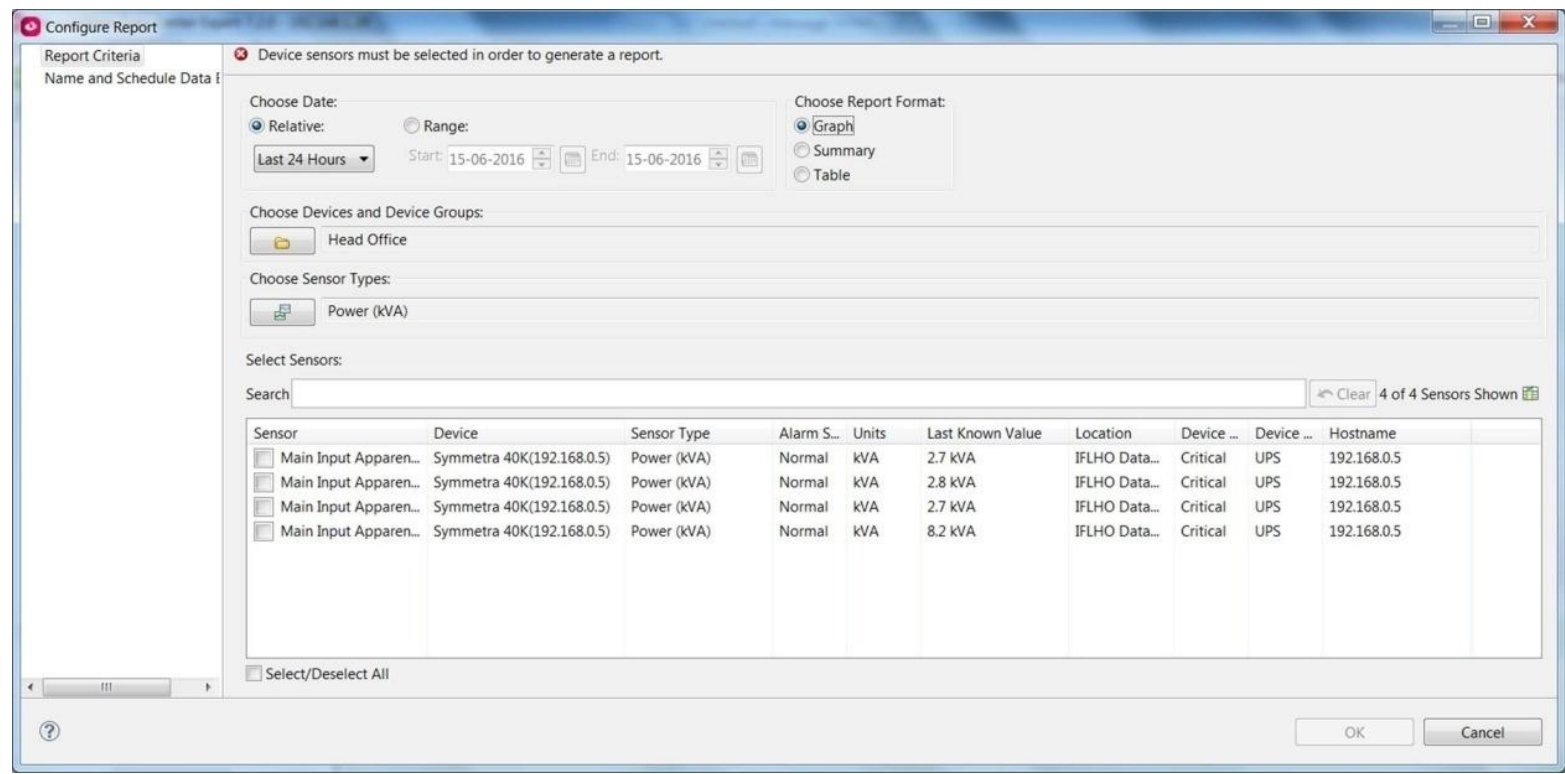

Fig. 3.2 Temprature analysis

The temprature of Rack 1 in data centeris 73.9 F OR 26.27C; The temprature of Rack 2 in data centeris 69.3 F OR 20.72C The temprature of Rack 3 in data centeris 74.3 F OR 23.5C; The temprature of Rack 10 in data centeris 71.8 F OR 22.12C The temprature of FF hall Rack in data centeris $67.5 \mathrm{~F}$ OR 19.72C

\subsection{Additional advanced energy-efficient actions}

There are extra measures, which can be taken keeping in mind the end goal to further expand the general productivity of the cooling frameworks supporting the server farm, including actualizing variable velocity/recurrence fan drives, using supply air control and expanding the chilled-water temperature where chilled-water frameworks are used.

\subsection{Implementing supply air control}

Supply air control ordinarily requires the expansion of supply air temperature sensors, as legacy aeration and cooling systems are normally not furnished with these gadgets. [16] Most legacy server farms are as yet using standard return air control systems. Be that as it may, server farms with supply air control strategies are acknowledging two essential advantages. In the first place, in conjunction with great wind current administration hones, supply air control ensures a predictable gulf air temperature through every single chilly path in the server farm. Second, it decreases the significance of the air handlers.

\subsection{Expanding Chilled Water Temperature}

A chilled water framework is one of the biggest single office force buyers in the server farm. Raising the air handler temperature set point gives the chance to expand the temperature of the chilled water and further diminish vitality utilization. Common legacy server farms have chilled water set focuses between $42-45^{\circ} \mathrm{F}\left(6-7^{\circ} \mathrm{C}\right)$. Offices that have experienced enhancement of their cooling frameworks have effectively raised their chilled water temperatures to $50^{\circ} \mathrm{F}$ $\left(10^{\circ} \mathrm{C}\right)$ or higher.

\subsection{Test case for energy consumption of UPS(symmetra PX 40) for data center}

This test case is established for measure the UPS power consumption in data center.The backup time of this UPS is 30 mint at $70 \%$ load .

\subsection{Strategies for increasing data center cooling efficiency}

3.8.1 Appropriate fixing of the server farm environment

A vapor seal assumes a basic part in controlling relative mugginess, lessening pointless humidification and dehumidification.

\subsubsection{Advancing wind current}

Rack course of action, PC room aeration and cooling system arrangement and link administration all effect the measure of vitality consumed to move air inside the basic office.

\subsubsection{Utilizing economizers where fitting}

Economizers permit outside air to be utilized to bolster server farm cooling amid colder months, making open doors for vitality free cooling.

\subsubsection{Expanding cooling framework proficiency}

New advancements, for example, variable limit frameworks and enhanced controls, are driving expanded proficiency of room ventilating frameworks.

\subsubsection{Conveying cooling nearer to the wellspring} of warmth

Supplemental cooling frameworks convey cooling nearer to the wellspring of warmth, decreasing the measure of vitality required for air development.

\subsection{Method for decreasing energy utilization in server farm}

This exploration will concentrate on the rest of the 50 percent that is devoured by vital emotionally supportive networks, for example, power, cooling and lighting (Figure 1). Alongside innovation frameworks themselves, the cooling framework devours the most vitality in the server farm, representing 37 percent of server farm power use.[17] Demands on cooling frameworks have expanded generously as of late as server densities have ascended to extraordinary levels. This change has not just made the requirement for expanded cooling 
framework limit, additionally has uncovered inefficiencies in existing ways to deal with server farm cooling about the same measure of force as the IT frameworks. On the off chance that power expenses were $\$ .10$ every hour, the aggregate yearly vitality costs for this office would be $\$ 5.25$ million ( $\$ 600 /$ hour $\mathrm{x} 8,765$ hours). A 10 percent lessening in the IT burden would make reserve funds of $\$ 1.05$ million, while a 30 percent change in cooling framework effectiveness would produce investment funds of around \$580,000.

Expand the Efficiency of Room

Ventilation systems

Three variables are basic to advancing the productivity of CRAC units:

- How effective the units work at incomplete burden.

- How productive the units are at expelling sensible warmth when contrasted with dormant warmth.

- How well numerous units cooperate.

Expanding Efficiency at Part Load

Server farms are composed with some level of cooling framework repetition. [18]Plus, the real Digital Scroll compressor innovation offers a more up to date approach to accurately coordinate limit and power utilization to the sought load and can convey essentially bring down vitality utilization contrasted with standard "altered limit" compressors.

\subsection{Method for saving time and money in the data center}

Estimated request arrival rate and denote the estimated service rate in the next adaptation window (i.e., over the next $\mathrm{W}$ time units)
1. Reduce time to lead reviews and look after stock. Whether every year, semi-yearly, month to month or week by week, utilizing innovation can speed review time permitting server farm staff to concentrate on higher-need undertakings.

2. Increase precision of server farm reviews. Utilizing a wand and portable truck is a less difficult, more exact technique for leading reviews and keeping up inventory. [19] It diminishes human blunder by evacuating the quantity of steps required in indexing resources. Data around a server farm resource is just entered once, as an advantage enters a server farm or amid the principal review. After that, the data dwells in a database.

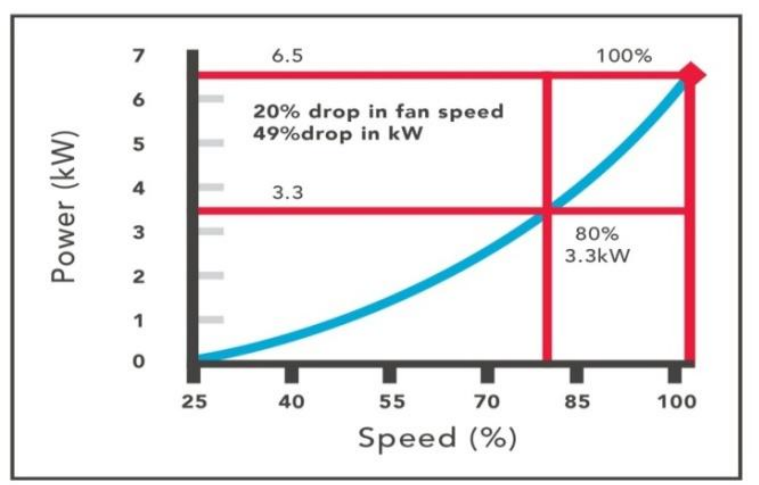

Fig. 3.3. The force utilization of a fan is a component of the solid shape of the fan speed.

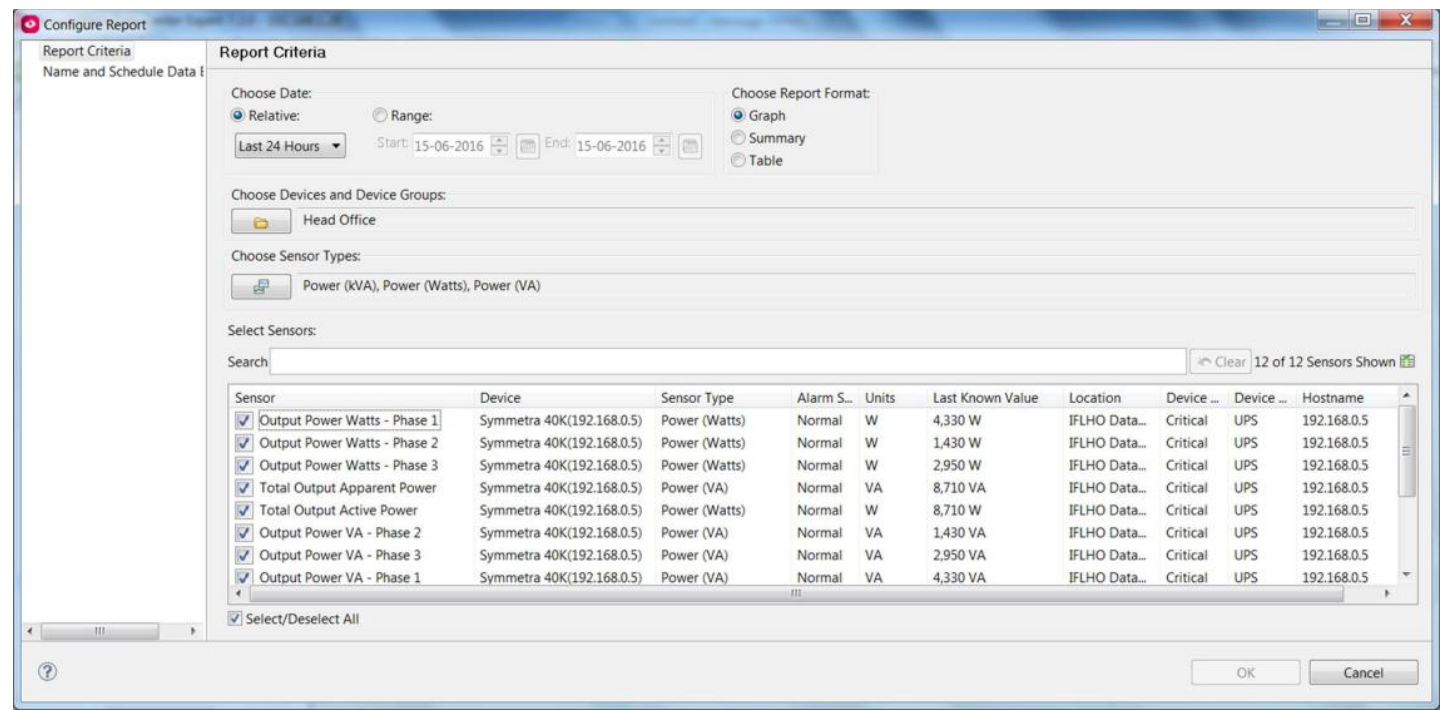

Fig. 3.4: Energy consumption of UPS

Phase 1: output power watts 4330; Phase 2: output power watts 1430; Phase 3 : output power watts 2950 total output apprent power 8710; total output active power 8710; Phase 1: output power VA 4330

Phase 2: output power VA 1430; Phase 3 : output power VA 2950; Total power consumption for cooling system in data center is $14 \mathrm{KW}$; Total power consumption of data center is $35 \mathrm{KW}$ 


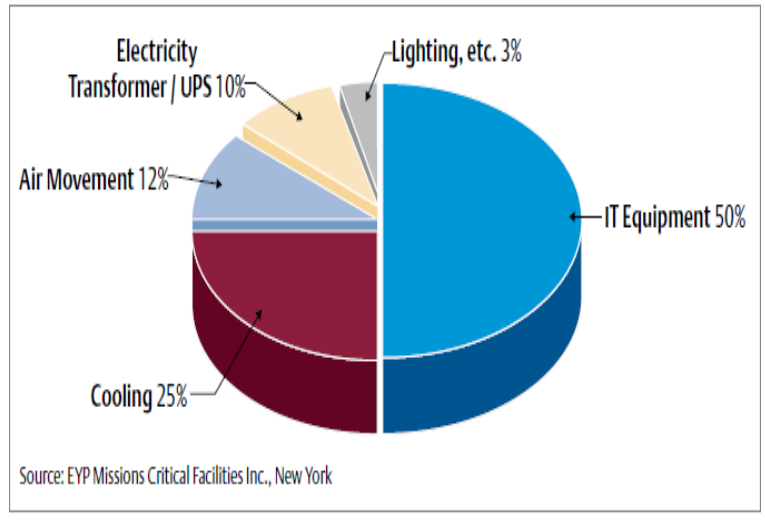

Fig. 3.5: Sources of data center energy consumption.

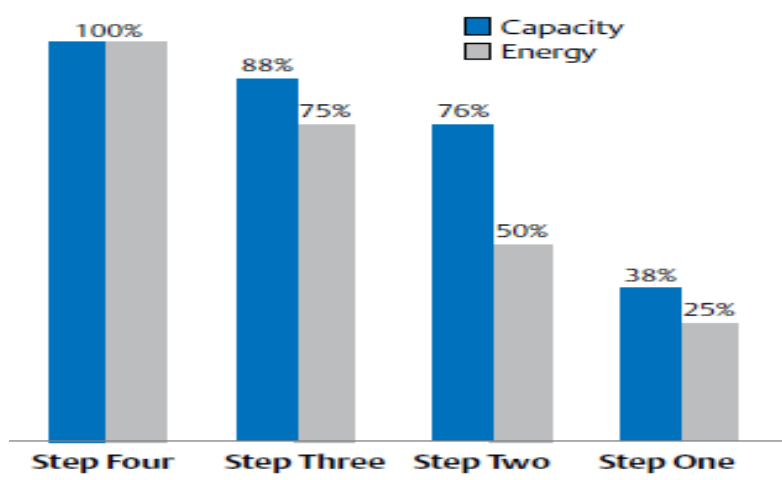

Fig. 3.6: Operating capacity versus energy consumption for the four stages of operation enabled by compressor unloading.

3. Quickly find basic server farm resources. The run of the mill 25,000 to 50,000 sq. ft. server farm may contain 5,000 to 15,000 individual resources - from racks, to servers to individual edges inside a server body. It is not unprecedented for an administration individual to enter a huge, venture server farm with an administration ticket and afterward spend the greater part of the day basically endeavoring to find the particular resource for repair.

4. Manage time-to-arrangement. Expansive server farms encounter almost 50 percent IT resource turnover because of hardware overhauls and end-of-life. A huge number of dollars in hardware touches base through the getting inlet, enters a holding confine in the server farm where it is arranged, and after that gets sent into racks on the server farm floor. [20]The longer an ideal opportunity to-send, the more these new resources cost the association. While numerous IT gear sellers now incorporate RFID labels specifically in their hardware, most server farms don't exploit this efficient innovation.

5. Manage administration level understandings (SLAs). Server farm experts can relate upkeep and guarantee data with each RFID gear tag. Along these lines they can screen and oversee hardware and related SLAs including when guarantees terminate, deterioration and transfer approaches. These can be looked into month to month, week by week or even day by day, helping a server farm tech all the more effortlessly screen, find and oversee IT resources

\subsection{Allocating resource shares to applications}

The assignment module is conjured intermittently (every adjustment window) to progressively parcel the asset limit among the different applications running on the mutual server. To catch the transient conduct of utilization workloads, it first presents a period area portrayal of an asset lining model. [21]This model is utilized to decide the asset prerequisites of an application in light of its normal workload and reaction time objective.

\section{RESULTS}

The expanding interest for capacity and calculation has driven the development of vast server farms - the monstrous server cultivates that run a large number of today's Internet and business applications. A server farm can contain numerous a great many servers and can use as much vitality as a little city. The gigantic measures of calculation force contained in these frameworks results in numerous intriguing conveyed frameworks and asset administration issues. In this proposal it is examine challenges identified with server farms, with a specific accentuation on how new virtualization advances can be utilized to streamline organization, enhance asset effectiveness, and lessen the expense of unwavering quality, all in application freethinker ways. it first study issues that identify with the underlying scope organization required while conveying applications into a virtualized server farm. it show how models of virtualization overheads can be used to precisely foresee the asset needs of virtualized applications, permitting them to be easily transitioned into a server farm. it next concentrate how memory closeness can be utilized to guide arrangement while adding virtual machines to a server farm, and show how memory sharing can be abused to lessen the memory impressions of virtual machines. This takes into consideration better server combination, lessening equipment and vitality costs inside the server farm. it then talk about how virtualization can be utilized to enhance the execution and proficiency of server farms using "live" movement and element asset allotment. It show mechanized, dynamic provisioning plans that can successfully react to the fast changes of Internet workloads without harming application execution. it then extend these movement devices to bolster consistently moving applications crosswise over low data transfer capacity Internet joins. At long last, it examine the unwavering quality difficulties confronted by server farms and present another replication strategy that permits distributed computing stages to offer superior, no information misfortune catastrophe recuperation administrations regardless of high system latencies.

\section{CONCLUSIONAND FUTURE WORK}

A server farm was an office used to house PC frameworks and related parts, for example, media communications and capacity frameworks. It for the most part incorporates repetitive or reinforcement power supplies, excess information correspondences associations, ecological controls (e.g., aerating and cooling, fire concealment) and different security gadgets. Substantial server farms are modern scale operations utilizing as much power as a residential community. Server farms have their roots in the tremendous PC rooms of the early periods of the processing business. Early PC frameworks, complex to work and keep up, required an uncommon domain in which to work. Numerous links were important to interface every one of the parts, and strategies to suit and arrange these were conceived, for example, standard racks to mount hardware, raised floors, and link plate (introduced overhead or under the hoisted floor). A solitary centralized server required a lot of force, and must be cooled 
to abstain from overheating. Security got to be critical PCs were costly, and was regularly utilized for military purposes. Essential outline rules for controlling access to the PC room were in this manner concocted.

Server farm modernization obliges associations to reexamine operational and authoritative needs, coming about because of changing financial patterns and new advancements. This diagram helps you fabricate a financially savvy and lightfooted operation that adjusts to business objectives. The significance of virtualization innovation being actualized in server farms to spare the expense and augment the effectiveness of various assets accessible. It proposed a five stage model to appropriately actualize virtualization. It begins by classifying servers and their related applications and assets into various asset pools. Consider that virtualization not just needs to portray the workloads that are wanted to be virtualized, additionally focus on the situations into which the workloads are to be connected. It is essential to decide the kind of servers their present status whether sit without moving or occupied, the amount of its expense to actualize server virtualization, the sort of innovation expected to accomplish the administration levels required lastly meet the security/protection goals.

\section{REFERENCES}

[1] Aamodt, A., and Plaza, E., 2011. Case-Based Reasoning, Advanced Micro Devices, Artificial Intelligence Communications, 7(1): 39-59.

[2] Ashrae, M., and Nelson, M., 2012. Thermal Guidelines for Data Processing Environments Mission Critical Facilities. American Society of Heating, Refrigerating, and Air- Applications, 16(6): 2-45.

[3] Basil, Y., 2012. Energy-Efficient Data Centers: A CloseCoupled Row Solution, International Journal of Computer Sciences (IJCSI) Journal of simulation model for Wireless technology, 2(5): 2-3.

[4] Bauer, R., 2011. Key Issues of a Formally Based Process Model for Security Engineering, In: Sixteenth International Conference "Software \& Systems Engineering \& Their, 15(2): 365-371.

[5] Bean, J., K. Dunlap, 2012. Energy-Efficient Data Centers: A Close-Coupled Row Solution, Computer Communication Review, 19(2): 32-48.

[6] Chen, A., Coskun, K., and Caramanis, M., 2013. Realtime power control of data centers for providing regulation service. In Decision and Control (CDC), 2013 IEEE 52nd Annual Conference on, 16(6): 2-4.

[7] Chen, M., Caramanis, C., and Coskun, K., 2014. The data center as a grid load stabilizer. In Design Automation Conference (ASP-DAC), 2014 19th Asia and South Pacific, 56(24): 105-112.

[8] Chiu, C., Stewart, A., and McManus, B., 2012. Electric grid balancing through low cost workload migration. SIGMETRICS Performance Evaluation Review, 40(3): $48-52$.

[9] Daim, S., Justice, J., and Krampits, M., 2011. Consumption in Data Centers, IEEE Communications Surveys\& Tutorials, 11(02): 345-876.

[10] Dhiman, T., Marchetti, G., and Rosing, T., 2009. A system for energy efficient computing in virtualized environments. In Proceedings of the 14th ACM/IEEE international symposium on Low power electronics and design (ISLPED), 32(12): 243-248.

[11] Elisa, P., Johnson, C., and Colby, W., 2011. Comparitive study of WLAN Wrox Suite, Computer Communication Review, 19(2): 32-48.

[12] Feldhaus, F., Freitag, S., and El Amrani, C., 2011. Stateof-the-ArtTechnologies for Large-Scale Computing, Large-Scale Computing Techniques for Complex System Simulations, Wiley-IEEE Computer Society, 16(5): 1-8.

[13] Gandhi, M., Balter, H., and Kozuch, A., 2012. Are sleep states effective in data centers? In IEEE IGCC, 12(6): 110.

[14] Ghatikar, W., Ganti, V., Matson, N., and Piette, M. A., 2012. Demand response opportunities and enabling technologies for data centers: Findings from field studies. 34(12):45-87.

[15] Hankendi, E., Reda, S., and Coskun, A. K., 2013. Adaptive power capping for virtualized servers. IEEE In ISLPED, 40(5): 415-420.

[16] Kant, K., 2013. Data Center Evolution A tutorial on state of the art issues and challenges, Computer Networks, 53(34): 345-543.

[17] Kumar, V., Talwar, P., Ranganathan, M., and Schwan, K., 2012. Coordinated management virtualized systems. Media Traffic, Journal of organizational Computing and Election Commerce, 15(4): 100-121.

[18] Lin, Z., Wierman, A., and Andrew, L., 2012. Online algorithms for geographical load balancing. In IEEE IGCC, 8(4): 1-10.

[19] Mell, P., and Grance, T., 2011. The NIST Definition of Cloud Computing\|, National Institute of Standards and Technology, Information Technology Laboratory, 15(2) 365-371.

[20] Mohammad, A., Talaat, A., Magdi, A., and Hoda, S., 2013, Congestion Control for Internet Newsletter, 31(4): 45-78.

[21] Zhenhuan, G., Xiaohui, G., and Wilkes, J., 2010. Predictive elastic resource scaling for cloud systems. In International Conference on Network and Service Management, 34(6): 9-16. 